\title{
RUDJER BOŠKOVIĆ INSTITUTE RADIOGARBON MEASUREMENTS X
}

DUŠAN SRDOČ, BOGOMIL, OBELIĆ, ADELA SLIEPČEVIĆ*, INES KRAJCAR BRONIĆ and NADA HORVATINČIĆ

Rudjer Bošković Institute, PO Box 1016, 41001 Zagreb, Yugoslavia

The following radiocarbon date list contains dates of samples measured since our previous list ( $\mathrm{R}, 1984$, v 26, no. 3, p 449-460). As before, age calculations are based on the Libby half-life $(5570 \pm 30)$ yr and reported in years before 1950 . The modern standard is 0.95 of the NBS oxalic acid activity. Sample pretreatment, combustion and counting technique are essentially the same as described in R, 1971, v 13, no. 1, p 135140 , supplemented by new techniques for groundwater processing ( $R$, 1979, v 21, no. 1, p 131-137).

Statistical processing of data has been computerized (Obelić \& Planinić, 1977; Obelić, 1980). Sample descriptions were prepared with collectors and submitters. The errors quoted correspond to $1 \sigma$ variation of sample net counting rate and do not include the uncertainty in ${ }^{14} \mathrm{C}$ half-life.

Calculations of age of speleothems and groundwaters are based on the initial activity equal to 0.85 of the NBS oxalic acid activity multiplied by 0.95 .

\section{ACKNOWLEDGMENTS}

We thank E Hernaus for preparation of samples and methane synthesis, A Turković for data processing and P Hojski for technical assistance.

\section{ARCHAEOLOGIC SAMPLES}

Yugoslavia

Croatia

\section{Vučedol series}

Systematic excavations at Eneolithic site Vučedol, "Strein's vineyard," near Danube R, Vukova $\left(45^{\circ} 21^{\prime} \mathrm{N}, 19^{\circ} 01^{\prime} \mathrm{E}\right), 110 \mathrm{~m}$ alt, E Croatia. Baden, Kostolac, and Vučedol cultural layers are dominant. Samples coll 1984 and 1985; subm by A Durman, Dept Archaeol, Univ Zagreb.

Z-1446. Vučedol, Quad 45, 1984

$4540 \pm 130$

Charcoal, Pit 47. Comment (AD): expected age: Baden phase.

Z-1617. Vučedol, Quad 69/99, 1985

$4500 \pm 100$ phase.

Charcoal, Pit 76 , depth $2.9 \mathrm{~m}$. Comment (AD): expected age: Baden

\footnotetext{
* Faculty of Veterinary Medicine, Physics Department, University of Zagreb
} 
Z-1618. Vučedol, Quad 131/161, 1984

$4300 \pm 100$ phase.

Charcoal, Pit 88, depth 4.2m. Comment (AD): expected age: Baden

Z-1619. Vučedol, Quad 86/87, 1985

$4400 \pm 100$ phase.

Charcoal, Pit 21, depth 2.6m. Comment (AD): expected age: Baden

Z-1447. Vučedol, Quad 115/145, 1984

$4290 \pm 120$ phase.

Charcoal, Pit 14, depth 2.5m. Comment (AD): expected age: Vučedol

Z-1449. Vučedol, Quad 107/138, 1984

$4190 \pm 120$ phase.

Charcoal, Grave 2, depth 2.3m. Comment (AD): expected age: Vučedol

Z-1453. Vučedol, Quad 107/138, 1984

$4290 \pm 120$ phase.

Charcoal, Grave 2, depth 2.9m. Comment (AD): expected age: Vučedol

Z-1454. Vučedol, Quad 107/138, 1984

$4130 \pm 120$ phase.

Charcoal, Grave 2, depth 3.2m. Comment (AD): expected age: Vučedol

Z-1621. Vučedol, Quad 62, 1985

$4300 \pm 100$ phase.

Charcoal, Pit 2, depth 3.6m. Comment (AD): expected age: Vučedol

Z-1622. Vučedol, Quad 36, 1985

$4100 \pm 100$ phase.

Charcoal, Pit 6, depth 2.3m. Comment (AD): expected age: Vučedol

\section{Z-1637. Vučedol, Quad 36, 1985}

$4300 \pm 100$

Charcoal from bottom of grave with 8 skeletons, Pit 6 , depth $4.7 \mathrm{~m}$. Comment (AD): expected age: Vučedol phase.

Z-1624. Vučedol, Quad 33/43, 1985

$4200 \pm 100$ phase.

Charcoal, Pit 19, depth 2.4m. Comment (AD): expected age: Vučedol

General Comment: ${ }^{14} \mathrm{C}$ ages older than classical chronology. Measurements at other sites of Baden culture made at Groningen and Berlin gave 4500 to $3900 \mathrm{yr}$ BP (classical chronology, 4250-3900 yr BP) and of Vučedol culture, 4300 to 4100 yr BP (classical chronology, 4100 to 3700 yr BP) (Dimitrijević, 1979).

\section{Sisak series}

Dry summer 1985 caused very low waters in Kupa $\mathrm{R}$ at Sisak $\left(45^{\circ} 29^{\prime} \mathrm{N}, 16^{\circ} 23^{\prime} \mathrm{E}\right), 99 \mathrm{~m}$ alt. Parts of wooden posts emerged from river 
bed, probably Roman bridge over Kupa R near ancient Siscia. Coll and subm 1985 by M Šmalcelj, Dept Archaeol, Univ Zagreb. Comment (MS): expected age: Roman period.

\section{Z-1580. Sisak No. 1}

$1830 \pm 100$

Tree rings 1 to 10 of larger sample of wood.

\section{Z-1581. Sisak No. 2}

$1880 \pm 100$

Tree rings 1 to 10 of smaller sample of wood.

General Comment: excellent agreement with expected age.

\section{Otišić series}

Charcoal mixed with ash from hearth, $0.6 \mathrm{~m}$ below ground level in karst depression Otišić near Sinj $\left(43^{\circ} 42^{\prime} \mathrm{N}, 16^{\circ} 14^{\prime} \mathrm{E}\right)$, alt $434 \mathrm{~m}$, S Croatia. Coll and subm 1984 by A Milošević, Regional Mus, Sinj. Comments (AM, DS): expected age: prehistoric settlement, 1900-1600 BP; dates indicate recent or medieval occupation of site.

Z-1451. Otišić No. 1

Z-1452. Otišić No. 2

\section{Z-1450. Zadar, Sv.Šime}

$$
\begin{array}{r}
330 \pm 120 \\
530 \pm 120 \\
1830 \pm 110
\end{array}
$$

Wooden board from wall above $\mathrm{N}$ colonnade, St Simeon church in Zadar $\left(44^{\circ} 07^{\prime} \mathrm{N}, 15^{\circ} 15^{\prime} \mathrm{E}\right)$, S Croatia. Medieval church was probably built on Roman foundation. Coll and subm 1984 by P Vežić, Inst Preservation Cultural Monuments, Zadar. Comment: date confirms Roman occupation of site.

Z-1566. Ц̆azma

$$
99.2 \pm 0.2 \% \text { modern }
$$

Modern

Fragments of wooden pipeline, depth 2.35 to $3.20 \mathrm{~m}$, Čazma $\left(45^{\circ} 45^{\prime} \mathrm{N}, 16^{\circ} 38^{\prime} \mathrm{E}\right)$. Coll and subm 1985 by V Štrk, Regional Mus Cazma. Comment (VS): expected age: late Middle Age. Comment: previously reported dates (Z-669: R, 1981, v 23, p 410) indicate replacement of destroyed wooden tubings with new ones.

\section{Sv. Ivan, Bol series}

Fragments of wooden beams and planks from St John's church in Bol, i Brač $\left(43^{\circ} 16^{\prime} \mathrm{N}, 16^{\circ} 40^{\prime} \mathrm{E}\right)$, S Croatia. Coll and subm 1985 by $\mathrm{G}$ Nikšić, Regional Inst for Preservation of Cultural Monuments, Split. Medieval church frequently reconstructed. Dates help to determine periods of reconstruction.

\section{Z-1540. Bol No. 1}

$550 \pm 100$

Wooden beam in SE corner supporting wall. Comment (GN): expected age: $10-12$ century AD. 
Z-1594. Bol No. 2

$320 \pm 100$

Wooden beam in SE corner supporting wall. Repeated measurement of Z-1540. Both dates are younger than expected.

Z-1541. Bol No. 3

$490 \pm 100$

Fragments of wooden shelf, morgue. Comment (GN): expected age: 15 century $\mathrm{AD}$ or later.

Z-1542. Bol No. 4

$103.9 \pm 0.9 \%$ modern

Fragment of wooden coffin mixed with earth in central grave.

\section{Jazvinački brig series}

Charcoal from Podvršje near Zadar ( $\left.44^{\circ} 07^{\prime} \mathrm{N}, 15^{\circ} 13^{\prime} \mathrm{E}\right)$, S Croatia. Coll and subm 1983 by Š Batović, Archaeol Mus, Zadar. Comment (SB): expected age: Bronze Age.

\section{Z-1265. Jazvinački brig No. 1}

$112.4 \pm 1.5 \%$ modern

Charcoal from depth 0 to $0.1 \mathrm{~m}$. Surface covered by grass with penetrating roots.

\section{Z-1264. Jazvinački brig No. 2}

$560 \pm 100$

Charcoal from depth 0.1 to $0.3 \mathrm{~m}$.

\section{Slovenia}

\section{Z-1466. Divje Babe}

$27,000 \pm 1300$

Cave bear bone (Ursus spelaeus), Divje Babe cave near Šebrelje, SW Slovenia. Coll and subm by D Brodar, Slov Acad Sci Arts. Sample from systematic excavations of Paleolithic site (Turk \& Dirjec, 1985). Bones buried in mixture of gravel and earth; cryoturbation was evident. Comment (DB): expected age: $>20,000 \mathrm{BP}$. Result agrees with previously dated charcoal (Z1032, -1033: R, 1984, v 26, p 450-451). Comment: dated on extracted and pyrolized collagen.

\section{Z-1582. Zamedvedca}

$10,500 \pm 200$

Fossil wood (Salix sp) in clay, Ljubljansko barje near Ljubljana $\left(46^{\circ} 00^{\prime} \mathrm{N}, 14^{\circ} 23^{\prime} \mathrm{E}\right), 300 \mathrm{~m}$ alt. Coll and subm 1985 by A Šercelj. Comment (AŠ): expected age: Pleistocene.

\section{Ajdovska jama cave series}

Charcoal from Ajdovska jama cave near Nemška Vas, Krško, E Slovenia. Coll 1983 and 1985 by T Bregant and subm by A Šercelj. Samples from systematic excavation of Neolithic site. Corresponds to earlier measurements, Z-1042 to -1045 (R, 1982, v 26, p 451). Comment (AŠ): expected age: Neolithic. 
Z-1178. Ajdovska jama No. 1

$5400 \pm 150$

Charcoal from right entrance to cave.

Z-1179. Ajdovska jama No. 3

$4700 \pm 200$

Charcoal from grave in cave.

Z-1554. Ajdovska jama No. 5

$4700 \pm 120$

Charcoal from central cave, depth $1 \mathrm{~m}$.

Z-1602. Ajdovska jama No. 68/85

$4850 \pm 130$

Charcoal from fireplace, depth $1.9 \mathrm{~m}$.

Z-1603. Ajdovska jama No. 34/85

$2900 \pm 120$ $2.3 \mathrm{~m}$.

Charcoal from fireplace in deepest layer with human bones, depth

\section{Moverna vas series}

Charcoal mixed with earth from hearth, Moverna vas near Črnomelj $\left(45^{\circ} 39^{\prime} \mathrm{N}, 15^{\circ} 13^{\prime} \mathrm{E}\right), 160 \mathrm{~m}$ alt, SE Slovenia. Coll 1984 by M Budja, Fac Sci Arts, Ljubljana, subm by A Sercelj. Dates Neolithic and Eneolithic cultures. Establishment of chronologic model of Neo-eneolithic of W Yugoslavia on basis of vertical stratigraphy and ${ }^{14} \mathrm{C}$ measurements. Comment (MB): expected age: 4000 to $5000 \mathrm{BP}$.

Z-1474. Moverna vas No. 1

$5400 \pm 140$

Charcoal, Layer 7, Quad 2, depth 2.2 to $2.5 \mathrm{~m}$.

Z-1475. Moverna vas No. 8

$4900 \pm 130$

Charcoal, Layer 6, Quad 2, depth 1.5 to $1.8 \mathrm{~m}$.

Z-1476. Moverna vas No. 9

$4050 \pm 120$

Charcoal, Layer 5, Quad 2, depth 1.5 to $1.8 \mathrm{~m}$. Comment (MB): result disagrees with other samples. Subm another sample (Z-1685) from same layer.

Z-1685. Moverna vas No. 11

$3900 \pm 100$

Charcoal, Layer 5, Quad 2, depth 1.5 to $1.8 \mathrm{~m}$ ( $c f$ Z-1476). Comment: both measurements on separate samples (Z-1476 and -1685) gave dates within statistical error.

\section{Donji Lakoš series}

Oakwood (Quercus robur) samples in soil, 1 to $2 \mathrm{~m}$ deep. Excavations of Bronze Age site near Donji Lakoš ( $\left.46^{\circ} 33^{\prime} \mathrm{N}, 16^{\circ} 26^{\prime} \mathrm{E}\right), 150 \mathrm{~m}$ alt. Coll by J Dular, Slov Acad Sci Arts; subm by A Šercelj, 1983. Comment (A ̌́): expected age: 3500 to $4000 \mathrm{yr} \mathrm{BP}$.

Z-1467. Donji Lakoš No. 9

$1120 \pm 100$ 
Z-1468. Donji Lakoš No. 10

Z-1469. Donji Lakoš No. 11

General Comment: results disagree with expected ages.

\section{Z-1421. Breg}

$6630 \pm 150$

Charcoal from hearth, 0.7 to $0.8 \mathrm{~m}$ deep in dolomite sand. Mesolithic site near Breg, Škoflijica $\left(45^{\circ} 59^{\prime} \mathrm{N}, 14^{\circ} 34^{\prime} \mathrm{E}\right), 294 \mathrm{~m}$ alt. Coll and subm 1984 by F Osole, Dept Geol \& Palaeontol, Univ Ljubljana. Comment (FO): expected age: $7000-8000 \mathrm{yr} \mathrm{BP}$.

\section{Ajdna series}

Charcoal with recent roots from Ajdna near Bled $\left(46^{\circ} 25^{\prime} \mathrm{N}\right.$, $14^{\circ} 07^{\prime} \mathrm{E}$ ), W Slovenia. Coll and subm 1985 by A Šrcelj. Comment (AŠ): expected age: Late Classical to Old Slavic times.

\section{Z-1575. Ajdna No. 1}

$109.9 \pm 0.7 \%$ modern

Small pieces of charcoal in soil.

Z-1576. Ajdna No. 2

Modern

Charcoal from hearth near ruins of church.

Neolithic in Serbia

Systematic excavations for study of early (Starčevo) and late Neolithic (Vinča) cultures in central Serbia were conducted at Grivac and Divostin as part of joint Yugoslav-American project organized by A McPherron, Dept Anthropol, Univ Pittsburgh, and D Srejović, Philos Fac, Univ Belgrade.

\section{Grivac series}

Charcoal from Neolithic settlement near Grivac $\left(44^{\circ} 01^{\prime} \mathrm{N}, 20^{\circ} 42^{\prime} \mathrm{E}\right)$, $18 \mathrm{~km} \mathrm{~W}$ of Kragujevac. Coll and subm 1984 by M Bogdanović, Regional Mus, Kragujevac. ${ }^{14} \mathrm{C}$ measurements made at Berlin gave $7250 \mathrm{yr} \mathrm{BP}$ for Starčevo and 6300 to 5930 yr BP for Vinča phase (McPherron \& Srejović, 1971).

\section{Z-1507. Grivac No. 1}

$5600 \pm 140$

Charcoal, Trench A, Layer V. Comment (AMcP): expected age: Vinča phase.

Z-1508. Grivac No. 2

$6000 \pm 140$

Charcoal, Trench A, Layer IX. Comment (AMcP): expected age: Starčevo phase.

Z-1505. Divostin D-5/2

$6900 \pm 150$

Charcoal, Layer VII, from systematic excavations near Divostin $\left(44^{\circ} 02^{\prime} \mathrm{N}, 20^{\circ} 50^{\prime} \mathrm{E}\right), 7 \mathrm{~km} \mathrm{~W}$ of Kragujevac. Coll and subm 1984 by $\mathrm{M}$ Bogdanović. ${ }^{14} \mathrm{C}$ measurements made at Berlin and British Mus gave 7100 
to 6900 BP for Starčevo phase. Charcoal, $35 \mathrm{~cm}$ below Level VII (Vinča phase) divided into 3 portions, dated at 3 labs (Bln-898: 5860 BP, BM-574: 5250 BP, Z-336: 6000 BP: R, 1977, v 19, p 472). Comment (AMcP): expected age: Starčevo phase.

Bosnia

\section{Z-1415. Pustopolje}

$3260 \pm 110$

Wooden plank from grave floor, Pustopolje near Kupres $\left(43^{\circ} 59^{\prime} \mathrm{N}\right.$, $\left.17^{\circ} 17^{\prime} \mathrm{E}\right), 1150 \mathrm{~m}$ alt. Plank buried in wet soil, $3.7 \mathrm{~m}$ deep. Coll and subm 1984 by A Benac, Acad Sci Arts B\&H, Sarajevo. Comment (AB): expected age: $3500 \mathrm{BP}$, based on assoc pottery.

\section{GEOLOGIC SAMPLES}

Fossil Wood Samples

Z-1317. Jelići

Fossil wood (unid. sp), Orašnica creek bed near Jelići, $4 \mathrm{~km}$ NE of Knin $\left(44^{\circ} 02^{\prime} \mathrm{N}, 16^{\circ} 11^{\prime} \mathrm{E}\right)$, Dalmatia, S Croatia. Coll and subm 1984 by A Pavičić, Geol Inst, Zagreb. Comment: unexpectedly well-preserved wood stumps in clayey Neogene layer. Date excludes recent growth of trees on much older soil.

\section{Z-1456. IKA No. 1}

$24,800 \pm 1000$

Fragment of wood (unid. sp) from layer of mud 5 to $6 \mathrm{~m}$ thick interspersed with wooden fragments, ca $100 \mathrm{~m}$ below sea level, at sea water depth 58m. Offshore drilling platform Panon, Adriatic Sea, Oilfield IKA, $60 \mathrm{~km} \mathrm{SW}$ of Pula $\left(46^{\circ} 08^{\prime} \mathrm{N}, 15^{\circ} 01^{\prime} \mathrm{E}\right)$, Istria, W Croatia. Coll and subm 1984 by I Muhovec, Geoexpert Co, Zagreb. Comment (IM): mud layer indicates silting from Po R, Italy.

\section{Z-1162. Petanjci}

$5790 \pm 160$

Fragments of wood from $4 \mathrm{~m}$ depth of Mura $\mathrm{R}$ alluvium at Petanjci near Radenci $\left(46^{\circ} 38^{\prime} \mathrm{N}, 16^{\circ} 02^{\prime} \mathrm{E}\right)$, NE Slovenia. Coll and subm 1983 by J Pezdič, Jožef Stefan Inst, Ljubljana. Comment (JP): dating of young river alluvium.

\section{Loess Samples}

Loess profiles dated for drafting of geologic map of Yugoslavia.

\section{Z-1455. Novi Sivac No. 2091}

$23,200 \pm 800$

Organic component of loess, open profile, depth $8 \mathrm{~m}$ near Novi Sivac, N Bačka $\left(45^{\circ} 21^{\prime} \mathrm{N}, 19^{\circ} 24^{\prime} \mathrm{E}\right)$, Vojvodina. Coll and subm 1984 by S Trifunović, Geol Inst, Belgrade.

Z-1444. Mikanovci No. 2194

$5860 \pm 150$

Calcareous concretions (loess kindchen) from silt layer above road near Mikanovci, Slavonia $\left(46^{\circ} 17^{\prime} \mathrm{N}, 18^{\circ} 33^{\prime} \mathrm{E}\right)$, E Croatia. Coll and subm 
1983 by M Brkić, Geol Inst Zagreb. Comment (MB): expected age: Würm III.

Z-1443. Prkovci No. 2329

$3850 \pm 130$

Calcareous concretions from loess, Prkovci, E Slavonia $\left(45^{\circ} 11^{\prime} \mathrm{N}\right.$, $\left.18^{\circ} 38^{\prime} \mathrm{E}\right)$, E Croatia.

\section{Koška series}

Calcareous concretions (loess kindchen) and snail shells from clayey silt, depth $1.5 \mathrm{~m}$, Koška near Našice $\left(45^{\circ} 32^{\prime} \mathrm{N}, 18^{\circ} 15^{\prime} \mathrm{E}\right)$, E Croatia. Coll and subm 1984 by B Korolija, Geol Inst Zagreb. Comments (BK,DS): expected age: younger Pleistocene to Holocene. Calcareous concretions of loess kindchen type show consistently younger age, indicating carbonaterich freshwater penetration in loess.

Z-1489. Koška, Na-7496

$4360 \pm 240$

Calcareous concretions (loess kindchen).

Z-1490. Koška, Na-7495

$17,400 \pm 500$

Land snail shells.

\section{Z-1481. Štitar No. 3449}

$2600 \pm 400$

Bones (unid. sp) from dark clay layer, depth $1.0 \mathrm{~m}$ below surface, Sava riverbank at Štitar near Županja $\left(45^{\circ} 05^{\prime} \mathrm{N}, 18^{\circ} 39^{\prime} \mathrm{E}\right)$, E Croatia. Coll and subm 1984 by M Brkić. Comment (MB): expected age: Holocene-Atlanticum.

\section{Other Samples}

Z-1161. Šratovci

Peat from alluvium of Mura $\mathrm{R}$ at Šratovci near Radenci $\left(46^{\circ} 38^{\prime} \mathrm{N}, 16^{\circ}\right.$ $02^{\prime}$ E), N Slovenia. Coll and subm 1983 by J Pezdič.

\section{Ribniško barje series}

Peat from peat bog Ribniško barje $\left(46^{\circ} 30^{\prime} \mathrm{N}, 15^{\circ} 17^{\prime} \mathrm{E}\right)$, alt 1550 , Pohorje Mt, N Slovenia. Coll and subm 1984 by A Šercelj, Slov Acad Sci Arts, Ljubljana. Dates help determine vegetation development periods and peat bog $\mathrm{fm}$.

Z-1365. 90-100cm $3100 \pm 120$

Z-1366. $190-200 \mathrm{~cm}$ $3590 \pm 120$ Z-1477. Cerna

Clay with black organic detritus, depth $6.5 \mathrm{~m}$. Clay deposit in brickyard Cerna, E Slavonia $\left(45^{\circ} 11^{\prime} \mathrm{N}, 18^{\circ} 41^{\prime} \mathrm{E}\right)$, E Croatia. No pollen grains found 
in sample. Coll and subm 1984 by M Brkić. Comment (MB): expected age: Quaternary marshy deposits.

\section{Slavonski Šamac series}

Clay with organic detritus and shells (unid. sp), Pleistocene profile, Sava R bank downstream from Slavonski Samac $\left(45^{\circ} 04^{\prime} \mathrm{N}, 18^{\circ} 30^{\prime} \mathrm{E}\right)$, E Croatia. Coll and subm 1984 by M Brkić. Comment: dates indicate organic material of secondary origin, probably roots.

Z-1479. Slavonski Šamac No. 2807 $4500 \pm 120$

Clay with organic detritus.

Z-1442. Slavonski Šamac No. 2807

$2340 \pm 110$

Charred wood embedded in clay.

\section{Z-1511. Radovna}

$$
\begin{aligned}
& >37,000 \\
& \delta^{13} C=+2.4 \% \text { o }
\end{aligned}
$$

River chalk deposit in Radovna $\mathrm{R}$ valley in vicinity of Lake Bled. Coll and subm by $\mathrm{J}$ Pezdič. Comment (JP): isotopic composition $\left({ }^{18} \mathrm{O}\right.$ and $\left.{ }^{13} \mathrm{C}\right)$ reveals detrital sediment deposited during postglacial period. Mineral composition: calcite $(60 \%)$, dolomite (30\%), other minerals $(10 \%)$.

\section{Čelarevo series}

Samples of peat, charred leaves, and organic detritus from several cores drilled at Celarevo near Bačka Palanka ( $\left.45^{\circ} 15^{\prime} \mathrm{N}, 19^{\circ} 24^{\prime} \mathrm{E}\right)$, W Vojvodina. Geologic survey of area near Danube R. Coll and subm 1985 by N Krstić, Geoinst, Belgrade. Comment (NK): expected age: Holocene.

\section{Z-1548. Čelarevo IB-36A, No. 1}

$1230 \pm 100$

Peat from sandy sediment, depth $2.3 \mathrm{~m}$.

Z-1549. Čelarevo IB-36A, No. 2

$530 \pm 100$

Organic mud from sandy sediment, depth $3.0 \mathrm{~m}$.

Z-1550. Čelarevo IB-36A, No. 3

$960 \pm 100$

Charred leaves, depth $4.3 \mathrm{~m}$.

Z-1551. Čelarevo IB-36A, No. 4

$26,200 \pm 1200$

Diagenetic mud, depth 22m.

Z-1591. Čelarevo G-1, No. 1

$$
740 \pm 100
$$

Peat, depth 2.6 to $2.65 \mathrm{~m}$, Borehole G-1. Comment (NK): expected age: younger Holocene (500-1200 yr).

Z1592. Čelarevo G-1, No. 2

$$
36,300+4400
$$

$-3600$

Peat, depth 20.7 to $20.8 \mathrm{~m}$, Borehole G-1. 
Peat depth 2.7 to $2.8 \mathrm{~m}$, Borehole G-2. Comment (NK): expected age: Holocene. Pollen analyses showed corn indicating layer younger than 350 yr.

Z-1598. Čelarevo G-2, No. 2

$8300 \pm 1000$

Peat, depth 22.5 to $22.6 \mathrm{~m}$, Borehole G-2.

Z-1599. Čelarevo G-3, No. 1

Peat, depth 1.85 to $1.95 \mathrm{~m}$. Borehole G-3.

Z-1600. Čelarevo G-3, No. 2

$560 \pm 100$

Peat, depth 2.4 to $2.5 \mathrm{~m}$, Borehole G-3.

Z-1601. Čelarevo G-3, No. 3

$650 \pm 100$

Peat, depth 2.9 to $2.95 \mathrm{~m}$, Borehole G-3.

\section{Z-1510. Lake Bled}

$39.2 \pm 0.5 \%$ modern

$\delta^{13} \mathrm{C}=-2 \%$

Lake chalk from Lake Bled $\left(44^{\circ} 22^{\prime} \mathrm{N}, 14^{\circ} 06^{\prime} \mathrm{E}\right)$, W Slovenia. Coll and subm by J Pezdič, Jožef Stefan Inst, Ljubljana. Comment (JP): chemically very pure calcite without admixture of detrital minerals formed by calcite precipitation from water (Dolenc et al, 1984). Comment: ${ }^{14} \mathrm{C}$ activity of sediment indicates ratio of biogenic $v$ inorganic carbon.

\section{HYDROGEOLOGIC SAMPLES}

\section{Croatia}

$3.3 \pm 0.3 \%$ modern

$31,800+2700$

Z-1503. Samobor No. 2

$-2200$

Water from artesian well at 190 to $220 \mathrm{~m}$ depth, Samobor $\left(45^{\circ} 48^{\prime} \mathrm{N}, 15^{\circ} 43^{\prime} \mathrm{E}\right)$, near Zagreb, W Croatia. Coll and subm May 1985 by N Tipić, INA-Naftaplin Co, Zagreb. Geothermal exploration near Zagreb.

\section{Podsused series}

Thermal ground water from borehole Podsused $\left(44^{\circ} 49^{\prime} \mathrm{N}, 15^{\circ} 50^{\prime} \mathrm{E}\right)$, W suburb of Zagreb. Coll and subm Aug 1985 by B Munda, INA-Proj Co, Zagreb.

\section{Z-1558. Podsused PDTE-1}

$6.4 \pm 0.4 \%$ modern

Thermal ground water, depth $600 \mathrm{~m}$. $\mathbf{2 0 , 7 0 0} \pm \mathbf{7 0 0}$

\section{Z-1559. Podsused PDT-1}

$11.1 \pm 0.4 \%$ modern

Thermal ground water, depth $282 \mathrm{~m}$. 
Slovenia

\section{Z-1174. Topolšica}

$$
\begin{array}{r}
3.0 \pm 0.3 \% \text { modern } \\
27,000 \pm 1800
\end{array}
$$

Water from borehole PC, Topolšica spa near Velenje $\left(46^{\circ} 24^{\prime} \mathrm{N}, 15^{\circ}\right.$ (0 1' E), N Slovenia. Tritium activity: $<0.2 \mathrm{~Bq} / \mathrm{l}$. Coll and subm May 1983 by M Veselič, Geol Inst Ljubljana.

\section{Z-1346. Medijske toplice}

$48.6 \pm 0.8 \%$ modern $4460 \pm 530$

Water from borehole V-3. Medijske toplice spa near Izlake $\left(43^{\circ} 42^{\prime} \mathrm{N}, 16^{\circ} 14^{\prime} \mathrm{E}\right)$, Central Slovenia. Coll and subm July 1984 by $\mathrm{M}$ Veselič.

\section{Kanižarica series}

Water samples from Kanižarica in Bela Krajina $\left(46^{\circ} 35^{\prime} \mathrm{N}, 15^{\circ} 03^{\prime} \mathrm{E}\right)$, E Slovenia. Coll and subm by P Kralj, Geol Inst, Ljubljana.

\section{Z-1405. Kanižarica No. 1}

$7.8 \pm 0.4 \%$ modern $19,000 \pm 600$

Water from borehole V-40. Coll Nov 1984. Tritium activity: $(0.4 \pm 0.3) \mathrm{Bq} / \mathrm{l}$.

\section{Z-1426. Kanižarica No. 2}

$13.2 \pm 0.4 \%$ modern $14,900 \pm 400$

Water from borehole V-41. Coll Dec 1984. Tritium activity: < 0.2 $\mathrm{Bq} / \mathrm{l}$.

\section{Z-1497. Kanižarica No. 3}

$16.0 \pm 0.4 \%$ modern $13,400 \pm 300$ $\mathrm{Bq} / \mathrm{l}$.

Water from borehole JV-2/82. Coll Apr 1984. Tritium activity: $<0.2$

\section{Z-1498. Kanižarica No. 4}

$34.1 \pm 0.5 \%$ modern $7500 \pm 200$

Water from borehole V-42/85. Coll Apr 1985. Tritium activity: $<0.2$ $\mathrm{Bq} / \mathrm{l}$.

$$
\begin{array}{r}
2.1 \pm 0.5 \% \text { modern } \\
31,900+2400 \\
-1600
\end{array}
$$

Z-1484. Kanižarica No. 5

Water from borehole JV-2/85. Coll Apr 1985.

\section{Bosnia}

\section{Tuzla series} $42^{\prime} \mathrm{E}$ ).

Water from several boreholes from salt mine Tuzla $\left(44^{\circ} 33^{\prime} \mathrm{N}, 18^{\circ}\right.$ 
Z-1300. Tuzla No. 1

$$
\begin{array}{r}
1.8 \pm 0.3 \% \text { modern } \\
30,800+2700 \\
-2100
\end{array}
$$

Water from borehole TD-22, depth 285 to $307 \mathrm{~m}$. Coll and subm May 1984 by N Djurić, Salt Mine Co, Tuzla.

\section{Z-1423. Tuzla No. 2}

$$
\begin{array}{r}
5.9 \pm \mathbf{0 . 3 \%} \text { modern } \\
\mathbf{2 1 , 3 0 0} \pm \mathbf{7 5 0}
\end{array}
$$

Water from borehole TD-23, 375m deep. Coll and subm Nov 1984 by $\mathrm{N}$ Djurić. Tritium activity: $<0.2 \mathrm{~Bq} / \mathrm{l}$.

\section{Z-1187. Tuzla No. 3}

$$
10.3 \pm 0.4 \% \text { modern }
$$$$
\mathbf{1 7 , 0 0 0} \pm \mathbf{5 0 0}
$$

Water from borehole TD-17, depth $480 \mathrm{~m}$. Coll and subm Nov 1983 by N Miošić. Tritium activity: $<0.2 \mathrm{~Bq} / \mathrm{l}$.

Z-1188. Tuzla No. 4

$$
33.8 \pm 0.5 \% \text { modern }
$$

$7400 \pm 200$

Water from spring TD-9 (“Tetima”). Coll and subm Nov 1983 by $\mathrm{N}$ Miošić. Tritium activity: $(0.6 \pm 0.2) \mathrm{Bq} / \mathrm{l}$.

\section{Z-1183. Gušića haus}

$$
\begin{array}{r}
13.0 \pm 0.4 \% \text { modern } \\
15,100 \pm 400
\end{array}
$$

Thermal water, Gornji Šeher near Banja Luka $\left(44^{\circ} 45^{\prime} \mathrm{N}, 17^{\circ} 45^{\prime} \mathrm{E}\right)$, NW Bosnia. Coll and subm Nov 1983 by N Miošić. Geothermal explorations near Banja Luka.

\section{Z-1186. Laktaši}

$$
\begin{array}{r}
4.4 \pm \mathbf{0 . 3 \%} \text { modern } \\
\mathbf{2 4 , 0 0 0} \pm \mathbf{1 0 0 0}
\end{array}
$$

Thermal water from Laktaši near Banja Luka $\left(44^{\circ} 45^{\prime} \mathrm{N}, 17^{\circ} 45^{\prime} \mathrm{E}\right)$, NW Bosnia. Coll and subm Nov 1983 by N Miošić. Tritium activity: $<0.2$ $\mathrm{Bq} / \mathrm{l}$.

\section{Z-1275. Banja Ilidža}

$$
\begin{array}{r}
1.4+0.3 \% \text { modern } \\
33,000+3500 \\
-2800
\end{array}
$$

Water from Ilidža spa near Sarajevo $\left(43^{\circ} 50^{\prime} \mathrm{N}, 18^{\circ} 20^{\prime} \mathrm{E}\right)$, Bosnia. Coll and subm Feb 1984 by N Miošić. Tritium activity: $<0.2 \mathrm{~Bq} / \mathrm{l}$.

\section{Z-1499. Fojnica}

$$
\begin{array}{r}
1.9 \pm 0.3 \% \text { modern } \\
30,500+2700 \\
-2300
\end{array}
$$

Thermal water from Fojnica spa, depth 50 to $300 \mathrm{~m},\left(43^{\circ} 58^{\prime} \mathrm{N}, 17^{\circ} 54^{\prime}\right.$ E), $670 \mathrm{~m}$ alt, central Bosnia. Coll and subm Apr 1984 by N Miošić. 
Dolenc, T, Pezdič, J, Mišić, J and Ogorelec, B, (ms) 1984, Isotopic and mineralogical characteristics of lake chalk in NW Slovenia: Paper presented at European regional mtg of sedimentology, 5th, Marseilles, France.

McPherron, A and Srejović, D, 1971, Early farming cultures in central Serbia (eastern Yugoslavia): Prelim rept, Natl Mus Kragujevac, p 1-26.

Obelić, B, 1980, Computer analysis and interpretation of radiocarbon data: Fizika, v 12, suppl $2, \mathrm{p} 139-161$.

Obelić, B and Planinić, J, 1977, Computer processing of radiocarbon and tritium data, in Povinec, $\mathrm{P}$ and Usačev, $\mathrm{S}$, eds, Internatl conf on low-radioactivity measurement and applications, Proc: The High Tatras, Slovenske pedagogicke nakladatelstvo, Bratislava, p 117 120 .

Srdoč, D, Breyer, B and Sliepčević, A, 1971, Rudjer Bošković Institute radiocarbon measurements I: Radiocarbon, v l3, no. 1, p 135-140.

Srdoč, D, Horvatinčić, N, Obelić, B and Sliepčević, A, 1982, Rudjer Bošković Institute radiocarbon measurements VII: Radiocarbon, v 24, no. 3, p 352-371.

Srdoč, D, Obelić, B, Horvatinčić, N, Krajcar Bronić, I and Sliepčević, A, 1984, Rudjer Bošković Institute radiocarbon measurements VIII: Radiocarbon, v 26, no. 3, p $449-$ 460 .

Srdoč, D, Sliepčević, A, Obelić, B and Horvatinčić, N, 1977, Rudjer Bošković Institute radiocarbon measurements IV: Radiocarbon, v 19, no. 3, p 465-475. 21, no.1, p 131-137. Bošković Institute radiocarbon measurements V: Radiocarbon, v

1981, Rudjer Bošković Institute radiocarbon measurements VI: Radiocarbon, v 23, no. 3, p 410-421.

Turk, I and Dirjec, J, 1985, Reka, Divje Babe I, Varstvo spomenikov: Ljubljana, v 27, p 189191. 